\title{
A self-consistent viscoplastic model: prediction of rolling textures of anisotropic polycrystals
}

\author{
R. A. Lebensohn and C. N. Tomé* \\ Instituto de Física Rosario (CONICET-UNR), 27 de Febrero 210 bis, 2000 Rosari (Argentina) \\ and Facultad de Ciencias Exactas, Ingeniería y Agrimensura (UNR), 27 de Febrero 210 bis, 2000 Rosario (Argentina)
}

\begin{abstract}
The plastic properties of anisotropic polycrystalline aggregates and polyphase materials are in general non-homogeneous and, as a consequence, so is the local plastic deformation. We present in this work a model that describes the plastic behaviour of non-homogeneous materials composed of anisotropic regions (grains or phases). Our model is based on describing each region as a viscoplastic inclusion embedded in the effective medium represented by the other grains, and incorporates explicitly the grain interaction with its surroundings and the plastic anisotropy of grain and matrix. Within the model the grain response is coupled to the overall response of the polycrystal and the grain deformation may differ from the polycrystal's. A characteristic of our approach is that those deformation systems with lower critical resolved shear stress tend to be more active, and less than five systems per grain are sufficient to accommodate the imposed overall deformation.

In this work we explore the consequences and the limits of the model, and its dependence on the assumed rate sensitivity as well. We combine the self-consistent formulation with a volume fraction transfer scheme for treating the reorientation due to twinning, and simulate rolling textures of brass (f.c.c.), Zircaloy (h.c.p.), calcite (trigonal) and uranium (orthorhombic). We compare the results with experimental measurements and Taylor-type predictions, infer information concerning the microscopic deformation mechanisms and discuss the limits of applicability of the approach.
\end{abstract}

\section{Introduction}

Plastic deformation of non-cubic materials is often characterized by a highly anisotropic behaviour of the constituent grains and by non-negligible twinning activity. The interaction of each grain with the surroundings is controlled by their relative anisotropies and dictates the amount of deformation that each one is going to accommodate. These features play an important role in the plastic response and have to be accounted for when modelling plastic deformation and texture development of polycrystals.

The classical Taylor-Bishop-Hill $[1,2]$ assumption of equal strain in every grain fulfils the compatibility condition but amounts to an extremely rigid interaction. The relaxed constraints (RC) [3] approach forces some of the stress components to be in equilibrium and relaxes the corresponding strain components. Its application, however, is restricted to materials with highly distorted grains. It seems logical that a more

\footnotetext{
*Permanent address: Reactor Materials Research Branch, AECL Research, Whiteshell Laboratories, Pinawa, R0E 1LO Man., Canada.
}

realistic formulation of texture development in plastically anisotropic materials must account for the fact that the grain-matrix interaction is neither completely rigid nor fully compliant. An important contribution in this respect is the viscoplastic self-consistent (VPSC) model proposed by Molinari et al. [4], which contemplates an interacting cluster of grains surrounded by a homogeneous anisotropic matrix.

As for twinning, it is a highly directional mechanism, which reorients whole fractions of the grain and is accompanied by a marked stress relaxation. A rigorous treatment of twinning should account for: twin nucleation and propagation; the associated stress relaxation; the twin morphology and its correlation with the parent lattice; and the reoriented twinning fractions. A treatment including all the afore-mentioned aspects is presently beyond our grasp and will require details of the grain microstructure to be incorporated. In any case, it can be safely stated that twinning complicates very much the modelling and the understanding of the polycrystal response. Even the relatively simple aspect of the twinning contribution to texture, which has been modelled in texture codes using approximate schemes that keep constant the number of orientations $[5,6]$, is 
not properly accounted for when twinning makes a substantial contribution to texture development.

In order to overcome some of the difficulties described above, we have proposed [7-9] a method for the exact treatment of twinning reorientation called the volume fraction transfer (VFT) scheme. We have also developed an anisotropic VPSC formulation [10] for dealing with grain interactions which is equivalent to the one-site case of Molinari et al. [4], though it improves upon the latter by making it easier to account for the matrix anisotropy in the calculations. Both the VFT scheme and the VPSC formulation have been successfully applied to the calculation of texture development in zirconium and zirconium alloys [7-10]. The aim of this paper is to extend such analysis to other anisotropic materials where twinning is a non-negligible deformation mode, and to discuss in some detail the assumptions inherent in the VFT and the VPSC methods.

The plan of the paper is as follows. In Section 2 we briefly review the main characteristics of the VFT scheme and the VPSC formulation. In Section 3 we present calculations of rolling textures for zirconium (hexagonal), brass (cubic), calcite (trigonal) and $\alpha-\mathrm{U}$ (orthorhombic), compare the predictions with the corresponding experimental textures and put forward a critical discussion of the results. Finally, in Section 4 we draw some general conclusions about the advantages and limitations of the models presented here.

\section{Modelling twinning and grain interaction in polycrystal codes}

\subsection{Twinning}

As we have shown $[8,9]$, treating the twinreoriented fractions with the VFT scheme leads to improved predictions of experimental texture features. Within the VFT scheme the polycrystal is represented by means of a discrete set of orientatins which are kept fixed while the associated volume fractions are allowed to evolve during deformation. The Euler space (Bunge angles convention) is partitioned regularly into equiaxed cells of $10^{\circ}$ by side. The orientations are made to coincide with the centre of the cells and a certain volume fraction of material is assigned to each cell corresponding to the initial texture. When plastic deformation is imposed on these representative grains, the resulting reorientation can be visualized as a displacement in Euler space of the point that represents the orientation. We assume that the cell as a whole displaces rigidly by the same amount and that the material is uniformly distributed within it. When displaced, the cell partially overlaps with the neighbouring cells and the volume fraction of material contained in the overlapped portions is subtracted from it and transferred to the neighbouring cells. This process of transference, repeated after every strain increment, leads to a gradual variation of the volume fraction in each cell and so to texture development. When twinning is active, the fraction of the grain that reorients is transferred to the corresponding non-neighbouring cell in Euler space and added to the fraction already assigned to the cell.

The VFT scheme described above is applicable to slip and twin reorientation. It permits us to account exactly for every twinned fraction in every twin system of every grain after each strain increment, without having to increase the initial number of orientations. Within the VFT scheme a grid orientation cannot be regarded as an individual grain, but is representative of all grains having similar orientations though not the same deformation history.

An aspect of twinning that is relevant to modelling refers to the notion of a critical resolved shear stress (CRSS) associated with twin activation. We analysed such an assumption in a recent paper [11] and concluded that a CRSS is a necessary requirement for twinning, although the activation may also depend upon other stress components in a manner that seems to be strongly related to the atomistic characteristics of the twin boundary. As a consequence, the CRSS has to be regarded as an effective value. Another aspect refers to the distinction between twiri nucleation and twin propagation. The high stress concentrations that drive twin nucleation arise because of a lack of favourably oriented slip systems in the grain. Since these stresses are most probably different from the uniform stress that we associate with each grain in our simulations, it is an implicit assumption that twins have somehow nucleated and that the CRSS is required to induce propagation. This interpretation helps to explain why the values of CRSS for twinning which are consistent with the observed plastic response are not much different from those associated with slip.

\subsection{VPSC formulation}

Accounting properly for the interaction of the grains with their surroundings is of utmost importance in any polycrystal model. The strength of such interaction dictates how much plastic deformation will accommodate the grain and how much the surroundings. It is evident that both the grain and the matrix plastic anisotropies will be directly responsible for such response and, as a consequence, both have to enter explicitly in the formulation. As discussed in the Introduction both the full constraints (FC) [1,2] and the RC [3] formulations do not formally account for grain interaction. However, the self-consistent approach that we use in this work is based on the assumption that each grain 
can be treated as an inhomogeneous inclusion embedded in the homogeneous effective medium (HEM) represented by the polycrystal. Such a formulation leads to an interaction equation that linearly relates stress and strain rate in the grain with the overall stress and strain rate of the effective medium. The condition that the average of stress and strain rate over all the grains has to be consistent with the equivalent macroscopic magnitudes makes for the selfconsistent resolution of the problem.

In what follows we present only the main equations of the formulation, which is described in detail by Lebensohn and Tomé [10]. The constitutive law for the grain is based on a rate-sensitive criterion which expresses the shear rate in each deformation system as a power of the resolved shear stress in such a system. Following Hutchinson [12], we write the strain rate in the grain as the kinematic sum of all potentially active systems

$$
\begin{aligned}
\dot{\varepsilon}_{i} & =\left[\dot{\gamma}_{11} \sum_{s=1}^{s} \frac{m_{i}^{s} m_{i}^{s}}{\tau_{\mathrm{c}}{ }^{s}}\left(\frac{m_{\mathrm{h}}^{\mathrm{s}} \sigma_{\mathrm{k}}}{\tau_{\mathrm{c}}{ }^{s}}\right)^{n-i}\right] \sigma_{i}^{\prime} \\
& =M_{i j}{ }^{\mathrm{c}(\mathrm{sec})}\left(\boldsymbol{\sigma}^{\prime}\right) \sigma_{j}^{\prime}
\end{aligned}
$$

where $\dot{\gamma}_{0}$ is a reference rate, $\tau_{\mathrm{c}}{ }^{\mathrm{s}}$ is a threshold stress and $n$ is the inverse of the rate sensitivity. The constitutive law eqn. (1) has already been expressed in a pseudolinear form where $\boldsymbol{M}^{\mathrm{c}^{(\mathrm{ec})}}$ is the secant viscoplastic compliance of the grain, giving the instantaneous relation between stress and strain rate. Except for $n=1, \boldsymbol{M}^{\mathrm{c}(\mathrm{sec})}$ depends on the applied stress, and the validity of the linear relation between stress and strain rate is limited to the precise point $\boldsymbol{\sigma}^{\prime}-\dot{\boldsymbol{\varepsilon}}$ that describes the grain state. The tangent compliance $\boldsymbol{M}^{\mathrm{c}(\mathrm{tg})}$ and the backextrapolated term $\dot{\boldsymbol{\varepsilon}}^{0}$ are defined by

$\dot{\varepsilon}_{i}=\left.\frac{\partial \dot{\varepsilon}_{i}}{\partial \sigma_{i}^{\prime}}\right|_{\sigma^{\prime}} \sigma_{j}^{\prime}+\dot{\varepsilon}_{i}^{0}=M_{i}^{\mathrm{ctlg}}\left(\boldsymbol{\sigma}^{\prime}\right) \boldsymbol{\sigma}_{j}^{\prime}+\dot{\varepsilon}_{i}^{\prime \prime}$

At the macroscopic level, the polycrystal response is assumed to be described by a pseudolinear constitutive equation similar to eqns. (1) and (2). If $\dot{\boldsymbol{E}}$ and $\boldsymbol{\Sigma}^{\prime}$ are the polycrystal strain rate and deviatoric stress respectively, the secant and tangent relations can be written as

$$
\begin{aligned}
& \dot{E}_{i}=M_{i j}{ }^{\prime \sec }\left(\boldsymbol{\Sigma}^{\prime}\right) \Sigma_{j}^{\prime} \\
& \dot{E}_{i}=M_{i j}{ }^{\prime \mathrm{tg} /}\left(\boldsymbol{\Sigma}^{\prime}\right) \Sigma_{j}{ }^{\prime}+\dot{E}_{i}^{\prime \prime}
\end{aligned}
$$

When $n$ is the same for all the systems, then the overall secant and tangent compliances are linearly related [12]

$$
\boldsymbol{M}^{(\mathrm{tg})}=n \boldsymbol{M}^{(\mathrm{sec})}
$$

and the same relation holds for the single-crystal compliances $\boldsymbol{M}^{\mathrm{c}}$.

Equation (4) amounts to regarding the aggregate as an HEM with a viscoplastic compliance $\boldsymbol{M}^{\text {it }}$ and a backextrapolated reference strain rate $\dot{\boldsymbol{E}}^{0}$, whose behaviour is identical to the overall behaviour of the polycrystal. Each grain, characterized by the viscoplastic compliance $\boldsymbol{M}^{\mathrm{c} \text { (tg) }}$ and a backextrapolated strain rate $\dot{\boldsymbol{\varepsilon}}^{\prime \prime}$, is in turn regarded as an inhomogeneous inclusion embedded in such an HEM. The problem of a viscoplastic inhomogeneity embedded in a homogeneous viscoplastic matrix being acted upon by a uniform stress $\boldsymbol{\Sigma}^{\prime}$ at infinity can be solved using the inclusion problem formalism $[10,13]$. Define the deviations of stress and strain rate in the inclusion with respect to the polycrystal averages as

$\dot{\varepsilon}_{k}=\dot{\varepsilon}_{h}-\dot{E}_{h}$

$\tilde{\sigma}_{1}{ }^{\prime}=\sigma_{1}{ }^{\prime}-\Sigma_{1}{ }^{\prime}$

The solution of the viscoplastic inclusion problem leads to the interaction equation

$\dot{\dot{\varepsilon}}=-\dot{\boldsymbol{M}}: \tilde{\sigma}^{\prime}$

where the colon indicates tensor contraction and the interaction tensor $\hat{\boldsymbol{M}}$ is defined as

$\tilde{\boldsymbol{M}}=(\boldsymbol{I}-\boldsymbol{S})^{-1}: \boldsymbol{S}: \boldsymbol{M}^{(\mathrm{tg})}=n(\boldsymbol{I}-\boldsymbol{S})^{-1}: \boldsymbol{S}: \boldsymbol{M}^{(\mathrm{sec})}$

Here $\boldsymbol{S}$ is the viscoplastic Eshelby tensor, a function of the shape of the inclusion and the tangent viscoplastic moduli, and the relation eqn. (5) was used to write the interaction tensor in terms of the secant compliance. The constitutive law eqn. (1) and the interaction equation (8) give ten non-linear equations for each grain, from which $\dot{\varepsilon}$ and $\boldsymbol{\sigma}^{\prime}$ can be derived.

So far, we have related the stress and the strain rate in each grain with the corresponding magnitudes in the HEM. In doing so we assume that the viscoplastic moduli of the grain and of the HEM are known in advance, which is not the case. Therefore a selfconsistent expression must be found from which the macroscopic modulus $\boldsymbol{M}^{(\mathrm{sec})}$ (and so the corresponding interaction matrix $\tilde{\boldsymbol{M}}$ ) can be calculated. Substituting eqns. (1) and (3) in eqn. (8) and using the condition that $\langle\dot{\boldsymbol{\varepsilon}}\rangle=\dot{\boldsymbol{E}}$ (where \langle\rangle indicates the weighted average over all the grains) leads to

$$
\boldsymbol{M}^{(\mathrm{sec})}=\left\langle\boldsymbol{M}^{(\mathrm{sec})}:\left(\boldsymbol{M}^{\mathrm{cisec}}+\tilde{\boldsymbol{M}}\right)^{-1}:\left(\boldsymbol{M}^{(\mathrm{sec})}+\tilde{\boldsymbol{M}}\right)\right\rangle
$$

The transcendental equation (10) gives the polycrystal compliance $\boldsymbol{M}$ and has to be added to the previous system in order to obtain an explicit solution of the problem. In practice, eqns. (1), (8) and (10) are not solved simultaneously for all the grains, but an iterative procedure is used, which is described in ref. 10. The procedure allows us to determine the stress in each grain, the grain's compliance tensor and the polycrystal compliance consistent with the latter and with the imposed strain rate $\dot{\boldsymbol{E}}$. The deformation of each grain is calculated assuming that such a strain rate acts during a time interval $\Delta t$. The grain reorientation as a conse- 
quence of such strain is calculated and the procedure is repeated until the final accumulated deformation is achieved.

Grain shape effects modify the grain-matrix interaction through the dependence of the Eshelby tensor upon the ellipsoid aspect ratios. They also induce a local rigid rotation of the ellipsoid and, as a consequence, affect the texture evolution. Both effects are important at high deformations, when the grains become more distorted [10]. Here we assume that all the grains undergo the same shape evolution, instead of keeping track of the individual grain shapes. The rationale behind this procedure is that the grain identity is not preserved within the VFT scheme: the volume fraction associated with each cell in Euler space results from adding up contributions from neighbour and twinning-related orientations that have undergone different deformation histories.

In what follows we discuss some interesting limit cases of the interaction eqn. (8) when the rate sensitivity exponent $n$ is increased. It is important, in this respect, to bear in mind that the secant compliances defined by eqns. (1) and (3) relate strain rate with stress in the grain and matrix and, as a consequence, they have to adopt finite values. Since the tangent tensor $\boldsymbol{M}^{\text {(tg) }}$ and the interaction tensor $\tilde{\boldsymbol{M}}$ depend linearly on $n$ (eqns. (5) and (9)), the stress deviations $\tilde{\sigma}$ must decrease when $n$ increases if the strain rate deviations $\tilde{\varepsilon}$ given by eqn. (8) are to remain bounded. This amounts to saying that when $n$ increases the matrix becomes more compliant, the grain-matrix interaction decreases, and the VPSC formulation predicts less deviation in stress from the average. This tendency starts to be appreciable for $n>20$ and seems to be an intrinsic limitation of the tangent assumption, which seems to be unsuitable to represent the highly non-linear response of the medium in the vicinity of the inclusion. This dependence of the interaction equation upon $n$ may also be regarded as an adjustable degree of freedom in the formulation (much the same as the empirical factor used by Berveiller and Zaoui [14] to "soften" the Kroner-Budiansky-Wu [15, 16] elastic interaction equation). In some cases (e.g. the brass deformation described later) one may want to take advantage of the previous feature and, based on physical considerations, increase the compliance of the matrix by using a high value of $n$. However, even for values of $n$ as large as 60 , such a procedure does not amount to a lower bound calculation: the stress deviation in the grains with respect to the average is about $6 \%$, an average of two systems are active in each grain, and the matrix still accommodates the relatively large strain misfit in an average sense.

Another interesting limit of the interaction equation is obtained when $n$ is eliminated from eqn. (9), which amounts to using the secant (instead of the tangent) compliance to calculate the interaction tensor $\tilde{M}$. Since the secant compliance is $n$ times smaller than the tangent compliance, this approximation amounts to considering a matrix $n$ times stiffer than what the formulation would require. As a consequence of such empirical modification the interaction tensor $\tilde{\boldsymbol{M}}$ remains small and "scales down" stress deviations in eqn. (8) to give very small strain rate deviations from the average. The latter result is akin to what would be obtained under FC conditions, with the difference that the deformation is not exactly the same for every grain.

As a conclusion, the VPSC formulation offers the possibility of tuning the compliance of the matrix by changing $n$ and by using either the tangent or the secant moduli to calculate the interaction tensor $\tilde{\boldsymbol{M}}$. This procedure amounts to "creating" a material with certain overall characteristics, and provides a powerful tool for relaxing the rather stringent Taylor or Sachs conditions, while still fulfilling equilibrium and compatibility in an average sense.

\section{Simulation of polycrystal rolling}

\subsection{Zircaloy-4 (hexagonal)}

Both the VFT scheme and the VPSC model were originally proposed to model the plastic response of zirconium alloys during rolling, swagging (uniaxial tension) and compression [8-10]. Here we present new predictions of texture development in cold-rolled Zircaloy- 4 which provide further insight into the crystallographic deformation mechanisms of this material. Charquet et al. [17] cold-rolled thin strips of Zircaloy-4 and obained two different textures, which they attribute to differences in the processing conditions of each batch of material. They label these textures as $\mathrm{T}$ and $\mathrm{L}$, depending on whether the predominant basal component is tilted (away from the normal direction (ND) of the sheet) towards the transverse direction (TD) or towards the rolling direction (RD) ("Laminage") respectively. However, they do not report the processing conditions and do not attempt to explain the difference in the resulting textures. In what follows, we investigate which combination of deformation modes leads to the observed textures.

According to the experimental evidence $[18-20]$, the following modes are found to be active at low temperatures in zirconium alloys: (a) $\{10 \overline{1} 1\}\langle 1 \overline{2} 10\rangle$ prismatic slip (to be denoted $\operatorname{pr}\langle a\rangle)$ is the most active one; (b) $\{10 \overline{1} 2\}\langle 10 \overline{1} 1\rangle$ tensile twinning (ttw) plays an important role; and $(\mathrm{c})\{21 \overline{1} 2\}\langle 21 \overline{1} \overline{3}\rangle$ compressive twinning (ctw) and/or $\{1011\}\langle 11 \overline{2} 3\rangle$ pyramidal slip $(\operatorname{pyr}\langle c+a\rangle)$ play a secondary role at low temperatures. In a previous work we were able to explain several deformation textures assuming the validity of the following set 
of CRSSs: (1) $\tau^{\mathrm{pr}(\mathrm{at})}=1.0, \tau^{\mathrm{tt \textrm {w }}}=1.25, \tau^{\mathrm{ct \textrm {w }}}=2.5[10]$. Here, we are also going to consider the set (2) $\tau^{\mathrm{pr}(\mathrm{a})}=1.0, \tau^{\mathrm{ttw}}=1.5, \tau^{\mathrm{pyr}(\mathrm{c}+\mathrm{a})}=4.0$. In both cases, $\operatorname{pr}\langle\mathrm{a}\rangle$ and ttw are the "soft" deformation modes while either ctw or pyr $\langle c+a\rangle$ is the "hard" complementary mode. A rate sensitivity exponent $n=19$ was used for the calculations that follow.

Deformation up to 1.0 true strain in rolling $(63 \%$ thickness reduction) was simulated using set 1 of CRSSs, while 0.6 true strain ( $45 \%$ thickness reduction) was simulated using set 2 . The corresponding basal and prismatic pole figures are reported in Fig. 1, where they are labelled $T$ texture and $L$ texture. The intensity of the basal poles along the ND-TD and the ND-RD directions are plotted in Fig. 2, superimposed on the experimental data of Charquet et al. [17]. From the comparison between experimental and calculated textures it seems evident that the $\mathrm{T}$ texture can be explained in terms of set 1 , although the calculated prism poles intensity in the RD is lower than the experimental value. The texture corresponding to set 2 , on the other hand, resembles the L texture, although the predicted maximum in the ND-RD plane is slightly shifted with respect to the experimental one. The

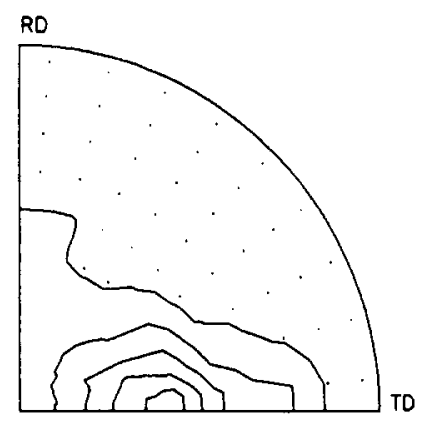

(a)

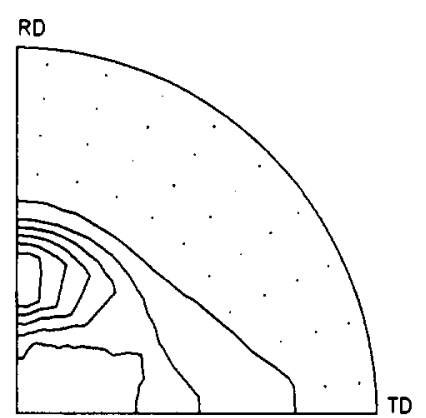

(c)

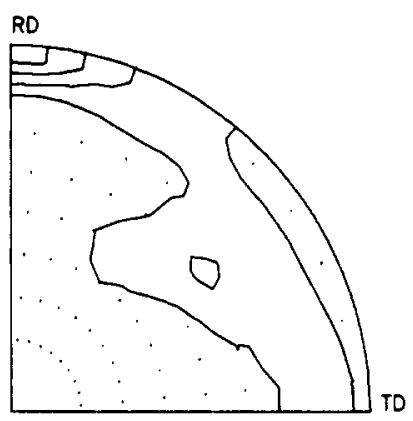

(b)

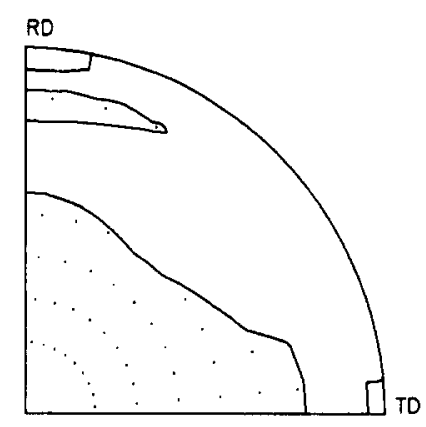

(d)

Fig. 1. (a) (0002) and (b) (1010) pole figures calculated with the VPSC formulation for 1.0 true strain using $\tau_{\mathrm{praj}}=1.0$, $\tau^{\mathrm{t \omega} w}=1.25, \tau^{\mathrm{ctw}}=2.5(\mathrm{~T}$ texture). (c) $(0002)$ and (d) (1010) pole figures calculated with the VPSC formulation for 0.6 true strain using $\tau^{\mathrm{pr}\langle\mathrm{a}\rangle}=1.0 \tau^{\mathrm{tww}}=1.5, \tau^{\mathrm{pyr}\langle\mathrm{c}+\mathrm{a}\rangle}=4.0$ (L texture). Equal-area projections are shown: $\longrightarrow$, multiples of random orientations; $\cdot$, intensities lower than 1.

agreement between predicted and experimental textures seems to indicate that tensile twinning was replaced by $\langle c+a\rangle$ slip in the L-type material. We speculate that the different thermomechanical treatment may have produced a smaller grain size in the L-type material, with the consequent suppression of twinning.

At this point, it is interesting to discuss the formation of the maxima in the basal pole figures, in terms of the slip and twinning activity. In Fig. 3 we plot the relative contribution of each mode to the total deformation. First, it is evident that most of the deformation is accommodated through the easy prismatic slip, which is a characteristic of the self-consistent approach. However, prismatic slip does not reorient the basal poles and the final texture has to be explained in terms of the other deformation modes. The maximum in the ND-TD plane predicted for the $T$ texture can be attributed to a combination of compressive twinning activity, which reorients the $c$ axes which are close to the ND towards the TD, followed by the activation of tensile twins, which tilts the $c$ axes towards their final stable position at $35^{\circ}$ from the ND, in the ND-TD plane. The observed maximum in the ND-RD plane for the $L$ texture can be explained in analogous terms: pyramidal slip tends to reorient the $c$ axis towards the $\mathrm{RD}[9]$, and tensile twinning provides the final reorientation, placing the $c$ axis on the ND-RD plane, close to the ND direction.

Figure 3 also shows the average number of active systems (AVACS) per grain as a function of the defor-
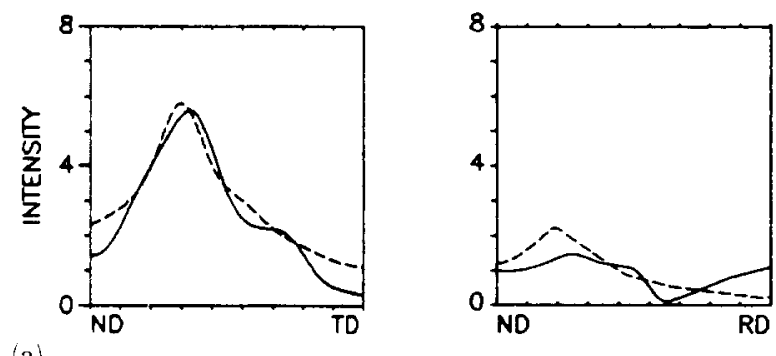

(a)
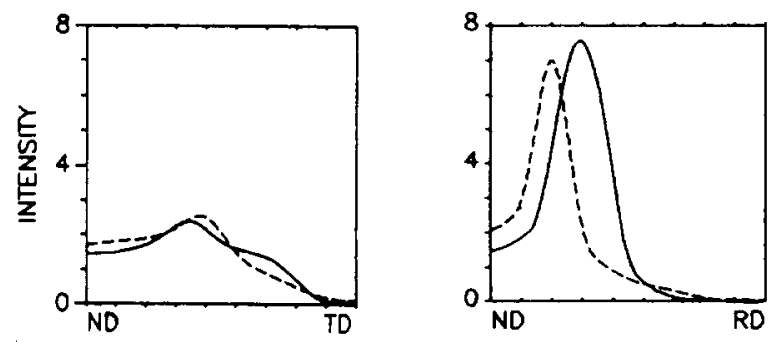

(b)

Fig. 2. Calculated intensities $(-)$ of $(0002)$ poles $v s$. tilt angle in the ND-TD and the ND-RD planes for (a) the T texture and $(b)$ the $\mathrm{L}$ texture. Experimental intensities reported in ref. 17 for Zircaloy- 2 are also plotted (-- -). 


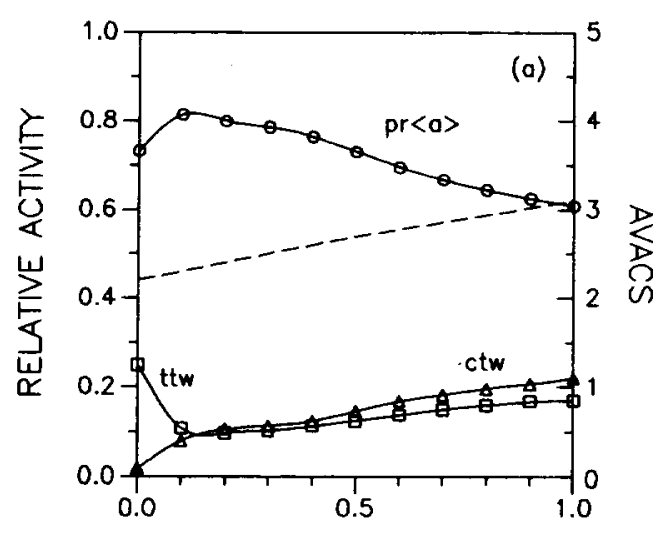

(a)

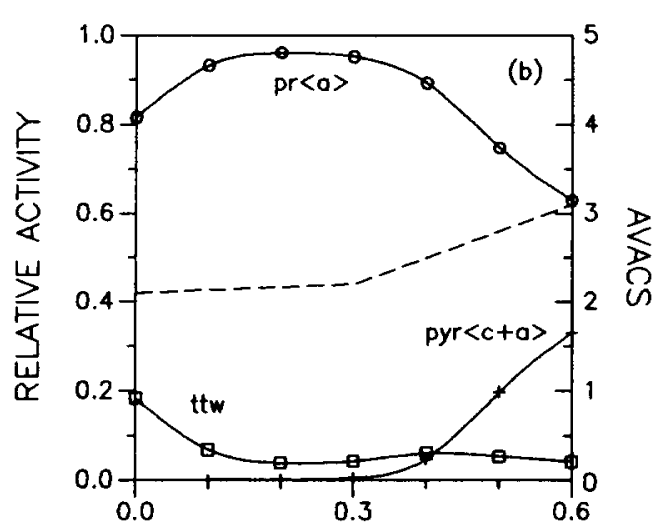

(b)

TRUE STRAIN

Fig. 3. Relative contribution of each mode to the total deformation and AVACS per grain (-- ) as a function of strain for (a) T texture and $(b) L$ texture.

mation. Here a system is considered to be active if the shear rate in it is at least $5 \%$ of the maximum shear rate in the grain. It can be seen that for both sets of CRSSs an average of about three active systems is required in each grain to accommodate the imposed deformation, with the largest contribution coming from the easy prismatc slip.

Another application of the VPSC scheme concerns the simulation of cross-rolling. Using set 1 of CRSSs, we simulated rolling up to 0.75 true strain, followed by a cross-rolling pass of 0.15 strain. The basal pole distribution that results is more symmetrically distributed around the ND of the sheet (see Fig. 4) and resembles the experimental texture reported by Ballinger for Zircaloy-2 [21]. The latter is also depicted in Fig. 4 for comparison purposes.

\subsection{Brass (cubic)}

Extensive work has been done to understand the microscopic mechanisms that dominate texture development of f.c.c. materials. The existence of two types of f.c.c. rolling texture is well established, each one corre-
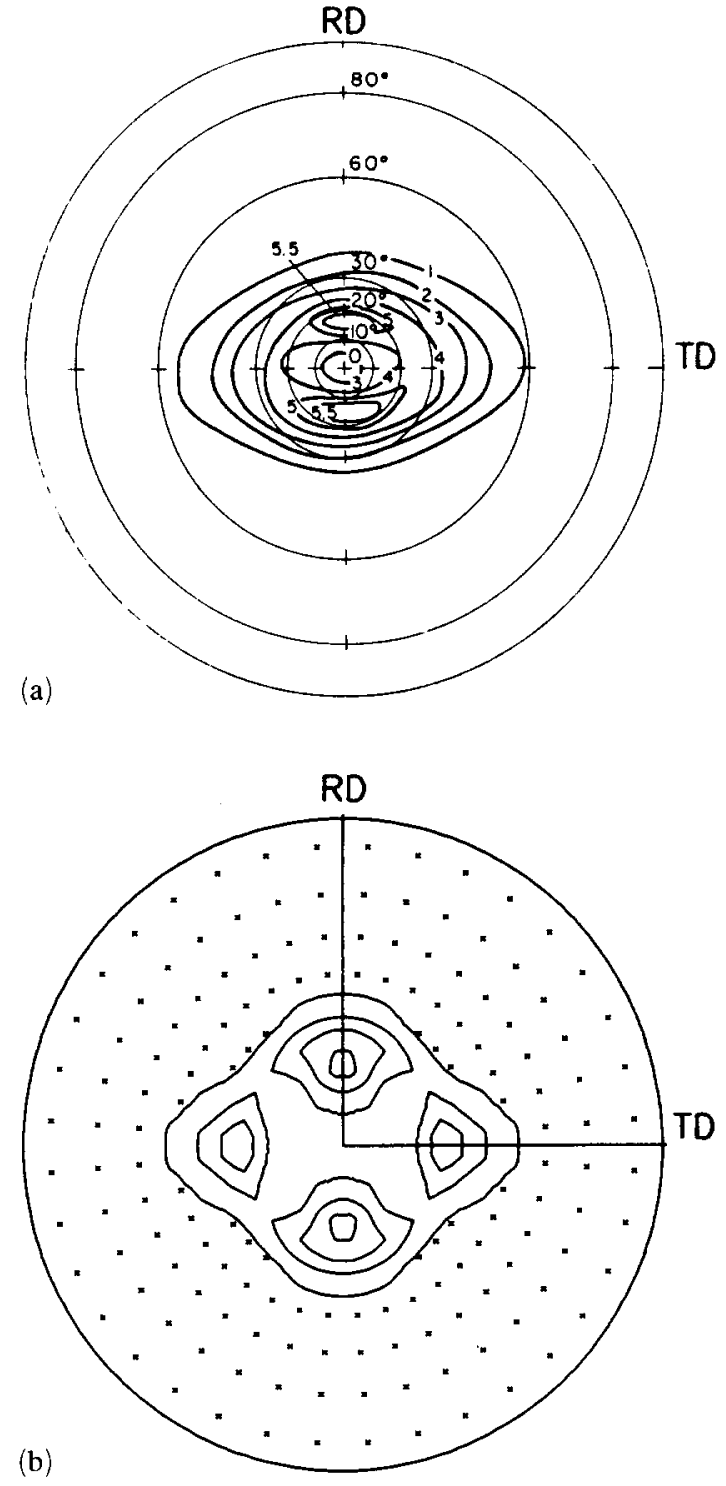

Fig. 4. (a) (0002) pole figure measured in cross-rolled Zircaloy-2 [21]. (b) Predicted (0002) pole figure after 0.75 rolling followed by 0.15 cross-rolling, using $\tau^{\mathrm{pr}\langle\mathrm{a}\rangle}=1.0, \tau^{\mathrm{tww}}=1.25, \tau^{\mathrm{ctw}}=2.5$. Stereographic projections are shown: - , multiples of random orientations; $\cdot$, intensities lower than 1.

sponding to materials with high or low stacking fault energy, known as copper texture and brass texture respectively. Here we will show how the VPSC scheme can be adapted to reproduce some of the observed microstructural features that are responsible for the brass texture.

The correlation between texture development and microstructure in rolled f.c.c. materials has recently been reviewed by Leffers and Jensen [22]. They point out that, in brass, $\{111\}\langle 11 \overline{2}\rangle$ twins form as very thin lamellae which are not homogeneously distributed but cluster to form bundles. the bundles exhibit a unique orientation in a given grain, parallel to one plane of the 
$\{111\}$ family. While the lamellae do not contribute appreciably to texture because they involve a small volume fraction of the grain, from the microstructural point of view they seem to play a major role in texture development. Grains containing this kind of cluster deform predominantly by $\{111\}\langle 1 \mathrm{i} 0\rangle$ slip on only one slip plane, namely the $\{111\}$ plane parallel to the clusters. A possible explanation is that the bundles are generated at the beginning of deformation, and that they influence the subsequent response of the grain. Within the framework of our model we assume uniform stress across the grains and we can only account for the microstructure in an "effective" way, through the parameters upon which the scheme depends. In this particular case the experimental evidence indicates that the tendency is for the grains to

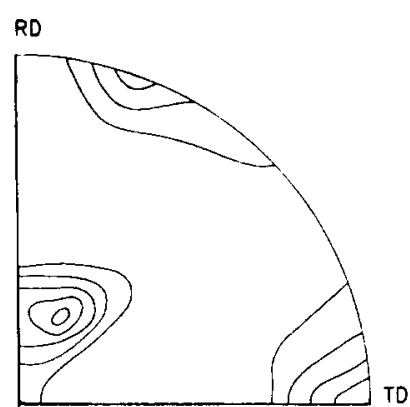

(a)

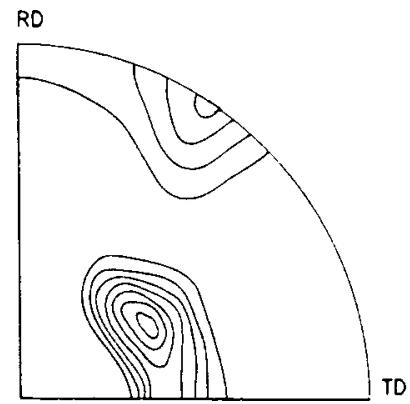

(b)

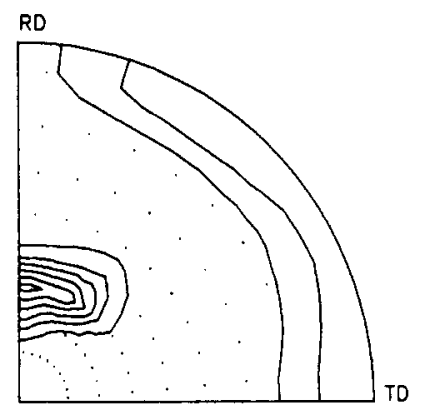

(c)

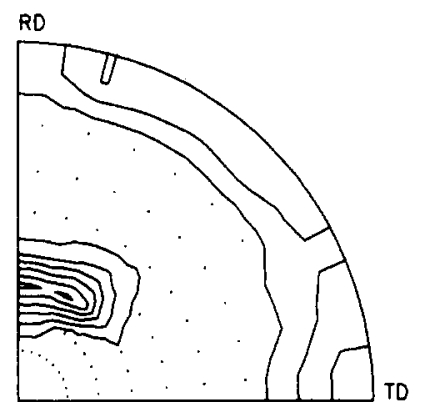

(e) accommodate deformation mainly through a conjugated slip-twin pair. This suggests, according to the discussion in Section 2, that we should use a high value of the rate sensitivity exponent $n$ in our calculations, in order to reduce the average number of systems required to accommodate the imposed deformation.

We simulated rolling of brass up to 2.0 true strain $(86 \%$ thickness reduction) assuming $\{111\}\langle 1 \overline{1} 0\rangle$ slip and $\{111\}\langle 112\rangle$ twinning as active deformation modes. The CRSSs were taken to be the same for both modes and exponents $n=19$ and $n=47$ were used. Figure 5 shows the corresponding $\{111\}$ and $\{200\}$ pole figures, together with typical brass experimental pole figures [23]. The texture obtained using $n=47$ shows a better agreement with the features of the experimental texture, but the main difference between the two cases is in the mode activity leading to such textures (Fig. 6). The AVACS is 3.2 for $n=19$, while for $n=47$ an average 2.1 systems per grain suffice to accommodate deformation. Besides, it is important to remark that in both cases the volumetric effect of twinning reorientation is not important: for $n=47$ and a strain increment of $1 \%$, the twinned volume fraction is $0.7 \%$ of the total

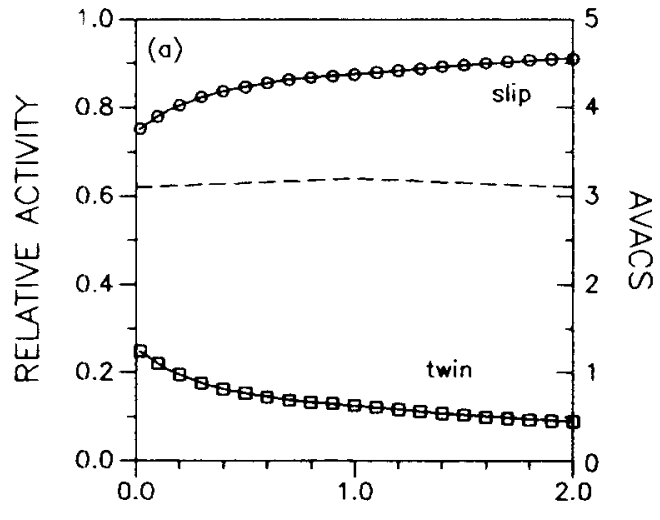

(a) TRUE STRAIN

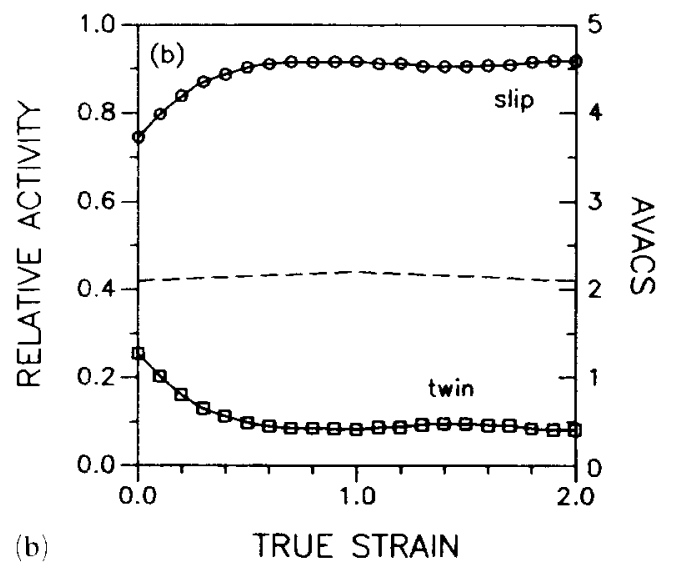

Fig. 6. Relative contribution of each mode to the total deformation and AVACS (-- ) as a function of strain, for the rolled brass case: (a) $n=19$; (b) $n=47$. 
volume in the first step, and decreases to $0.25 \%$ for the last deformation step.

A more revealing statistic is obtained for the case $n=47$ if one discriminates between the combinations of active systems per grain, where a system is considered to be active if its shear strain is at least $5 \%$ of the maximum shear strain in the grain. Over a total of 2916 orientations, in about $60 \%$ of them there is either one slip, or one twin, or two slip, or two twinning systems active, throughout deformation. In the remaining $40 \%$ of orientations, one slip and one twinning system are active simultaneously. Of those, $60 \%$ are conjugated pairs in the same shear plane, although only $25 \%$ should be found if all the slip-twin combinations were equally probable. Such correlation seems to be in qualitative agreement with the experimental observation, and suggests that the lower matrix stiffness associated with the VPSC method when $n$ is large provides a valid representation of the plastic response of materials with low stacking fault energy. Similar results were obtained by Leffers and Jensen using a modified Sachs model [22].

\subsection{Calcite (trigonal)}

Calcite is a material with trigonal crystal structure, and whose plastic behaviour is relevant to geological applications. Calcite is an appropriate material for testing both the VFT scheme and the VPSC formulation, because of its high plastic anisotropy and because it twins profusely at room temperature. The active deformation modes in calcite are: $\{01 \overline{1} 8\}\langle 0 \overline{4} 41\rangle$ twinning (to be denoted as $\left.\mathrm{e}^{+}\right),\{10 \overline{1} 4\}\langle 20 \overline{2} \overline{1}\rangle$ slip $\left(\mathrm{r}^{-}\right.$, while the opposite systems are denoted $\left.\mathrm{r}^{+}\right)$and $\{01 \overline{1} 2\}\langle 20 \hat{2} \overline{1}\rangle \operatorname{slip}\left(\mathrm{f}^{-}\right)$. Figures $7(\mathrm{a})$ and $7(\mathrm{~b})$ show two experimental textures of calcite deformed in plane strain compression up to 0.5 true strain $(40 \%$ finite shortening), measured at $100{ }^{\circ} \mathrm{C}$ and $400{ }^{\circ} \mathrm{C}$ respectively [24]. Calcite presents a non-negligible strain rate sensitivity and for the purpose of the calculation we assumed an exponent $n=9$ and two sets of CRSSs: (1) (a)
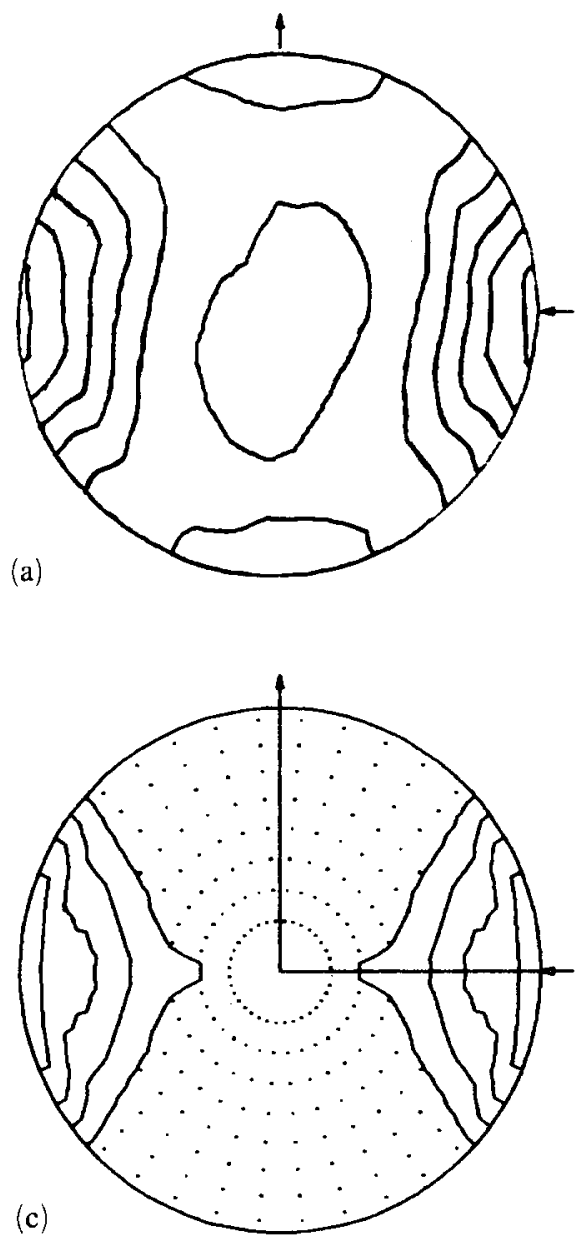

(b)
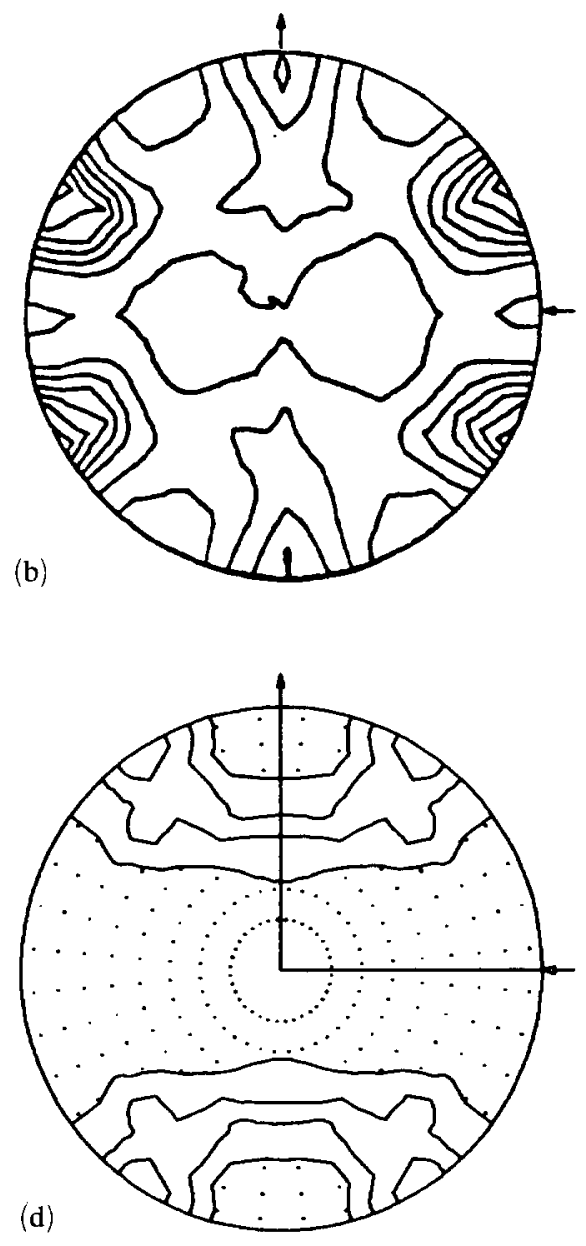

Fig. 7. (0002) experimental pole figures for calcite deformed in pure shear [24] at (a) $100{ }^{\circ} \mathrm{C}$ and (b) $400{ }^{\circ} \mathrm{C}$. (c) (0002) pole figures predicted after 0.5 true strain using CRSSs 1: $\tau^{r^{+}}=1.5, \tau^{\tau^{+}}=1.0, \tau^{e^{+}}=0.2$. (d) Same using CRSSs $2: \tau^{r^{-}}=1.5, \tau^{r^{+}}=1.0, \tau^{f^{f^{-}}}=1.0$, $\tau^{e^{+}}=0.2$. Equal-area projections are shown: - multiples of random orientations; ${ }^{\prime}$, intensities lower than 1. 


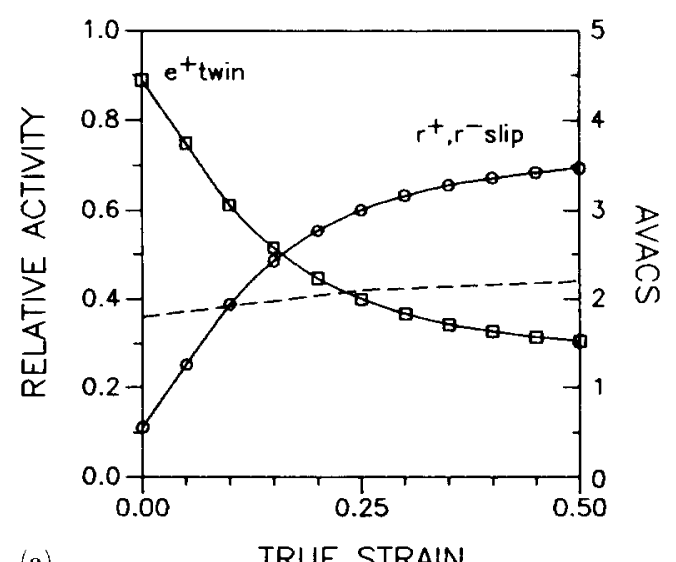

(a)

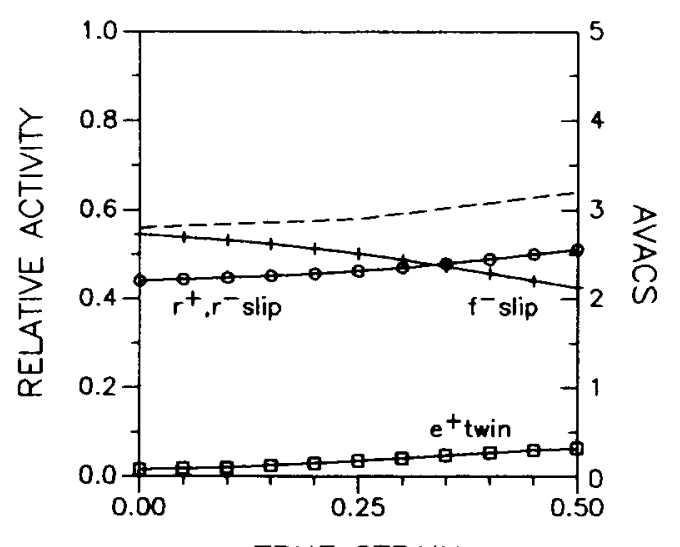

(b)

TRUE STRAIN

Fig. 8. Relative contribution of each mode to the total deformation and AVACS (---) as a function of strain, for the calcite case: (a) CRSSs 1; (b) CRSSs 2.

$\tau^{\mathrm{r}^{-}}=1.0, \quad \tau^{\mathrm{r}^{-}}=1.5, \quad \tau^{\mathrm{e}^{+}}=0.2 ;$ (2) $\tau^{\mathrm{r}^{-}}=1.0, \quad \tau^{\mathrm{r}^{+}}=1.5$, $\tau^{f^{-}}=1.0, \tau^{\mathrm{e}^{+}}=3.0$. The predicted textures are plotted in Figs. $7(\mathrm{c})$ and $7(\mathrm{~d})$, while the relative system activity and the AVACS are depicted in Fig. 8. It can be seen that the substantial differences in the experimental textures at different temperatures can be explained in terms of a hardening of the $\mathrm{e}^{+}$twinning and its replacement by $\mathrm{f}^{-}$slip at high temperatures. These results are similar to those predicted using a Taylor approach [24] and an "isotropic" self-consistent approach [6]. We attribute the similarity between the predictions of different models to the scarcity of deformation modes present in each grain which, by reducing the choice of active systems, leads to similar reorientations in all the cases. However, the difference between the VPSC and the FC approaches is the system activity leading to those textures. Within the VPSC formulation fewer systems are required and the softer systems contribute more to deformation (see Fig. 8).

\section{4. $\alpha-U$ (orthorhombic)}

Uranium is a low symmetry material (orthorhombic) with highly anisotropic thermomechanical properties.
It deforms through a variety of slip and twinning systems [25], which greatly complicates the interpretation of the experimental textures and the theoretical modelling. The analysis of the topological domains of CRSSs, which proved to be very useful in linking active systems with associated textures in hexagonal [9] and trigonal materials [24], is less effective in this case. We have tried several combinations of systems and CRSSs but a systematic study of the plastic characteristics of this material would be required which is still lacking. Besides the rolling simulations done by Morris [26], texture development of $\alpha$-U along different strain paths has been calculated by Rollett using an FC code in combination with a VFT scheme for treating the twinning reorientation [27]. The set of deformation modes and CRSSs used in ref. 27 are: $\tau^{(010) \mid 10 \%(s) \text { sip }}=0.5$,

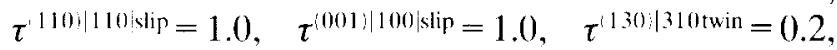
$\tau^{172 \mid 312 \text { twin }}=1.0, \quad \tau^{1112: 372 \mid \text { twin }}=1.0, \quad \tau^{1 / 47 \mid 512 \text { twin }}=2.5$. Here we simulate rolling up to 2.0 true strain, using the same set of CRSSs and the VPSC formulation. We compare our results against experimentally measured textures [26] and the predictions of the FC scheme. The results are reported in Fig. 9 in the form of $(001)$, $(010)$ and $(100)$ pole figures. It can be seen that both predicted textures reproduce some of the experimental features, but neither the FC nor the VPSC formulation predicts the maxima corresponding to the texture components (201)[010] and (263) [310].

As in the calcite case, the final FC and VPSC textures are not substantially different from each other, but the relative activity of the deformation modes is, as can be judged from Fig. 10. In the FC case, the three hardest twinning modes (specially $(197)\langle 512\rangle$, together with $(112)\langle 372\rangle$ and $(172)\langle 312\rangle)$ are more active than the soft twinning mode $(130)\langle 310\rangle$. In the VPSC calculation, in contrast, the higher activity corresponds to the slip systems, and the twinning modes play a less relevant role, with the exception of the soft $(130)\langle 310\rangle$ at the early stages of deformation.

\section{Conclusions}

We present in this work a comprehensive anisotropic VPSC formulation and apply it to the calculation of rolling textures in materials with different symmetries and plastic characteristics. Since all of the materials considered present twinning, we combine the self-consistent formulation with a VFT scheme. In all the cases the predicted textures compare well with the experimental ones. For Zircaloy, in particular, we are able to interpret qualitative differences in experimental textures in terms of different deformation systems being active. This shows how ths method can be used 


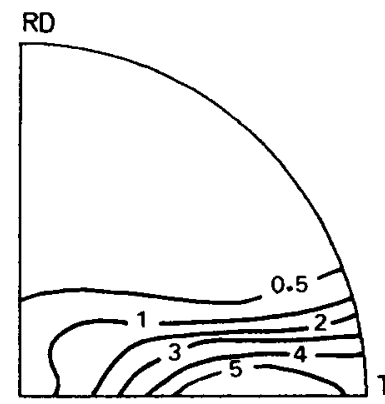

(a)

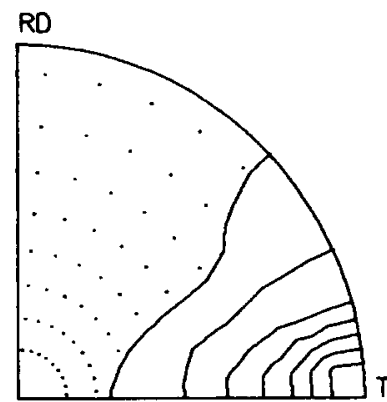

(d)

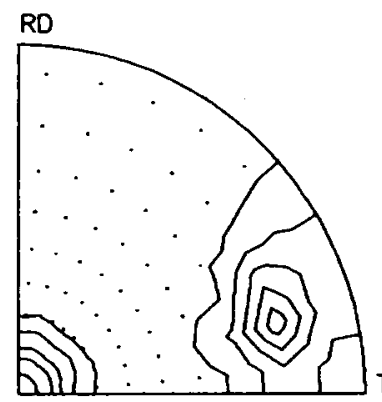

(g)

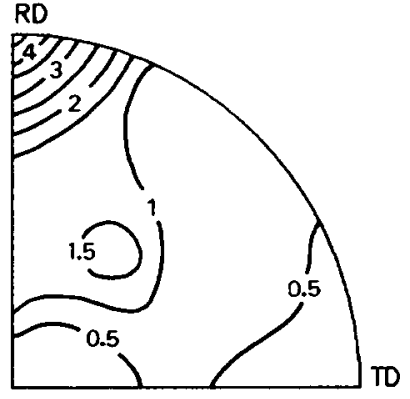

(b)

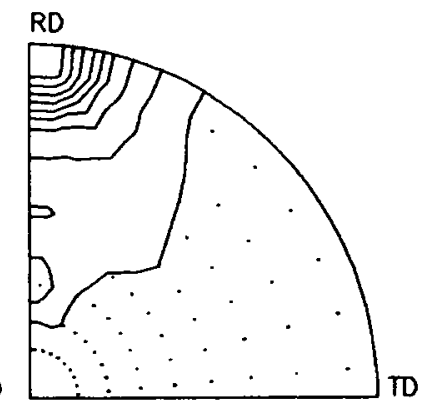

(e)

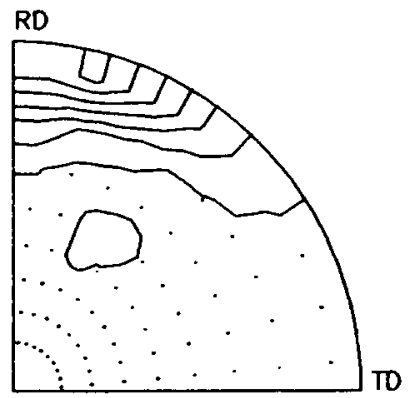

(h)

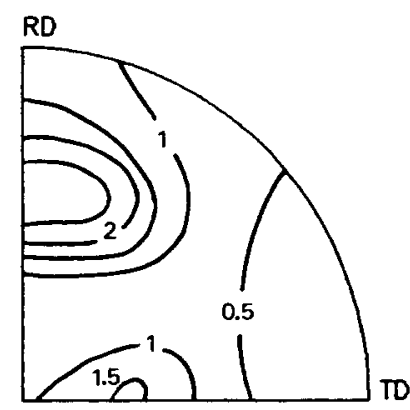

(c)

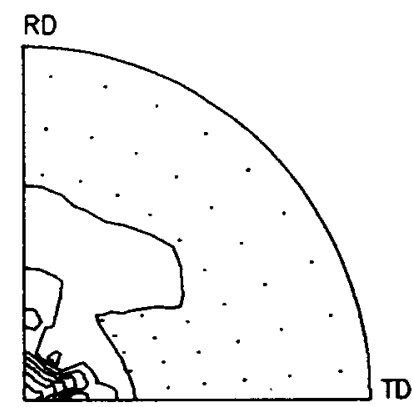

(f)

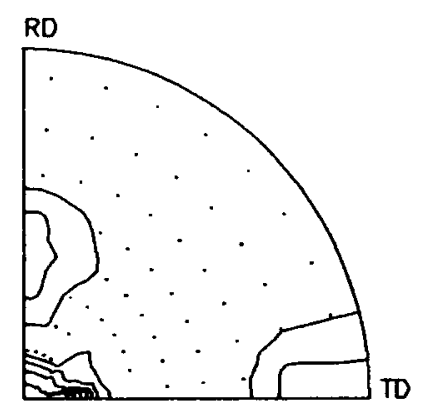

(i)

Fig. 9. (a) $(001),($ b) $(010)$ and (c) (100) pole figures measured in rolled $\alpha-U$ [26]. (d) (001), (e) (010) and (f) $(100)$ pole figures calculated with the FC formulation after 2.0 true strain using $\tau^{(010)[100) \text { slip }}=0.5, \tau^{(110)[119) \text { slip }}=1.0, \tau^{(001)[100] \text { slip }}=1.0, \tau^{(130) / 310] \text { twin }}=0.2$, $\tau^{(172) \mid 312] \text { twin }}=1.0, \tau^{(112) \mid 372\} \text { twin }}=1.0, \tau^{(197) \mid[12 \mid \text { twin }}=2.5$. (g), (h), (i) Same with the VPSC formulation. Stereographic projections are shown: - multiples of random orientations; $\cdot$, intensities lower than 1.

to infer information about the microstructural parameters.

We stress in this work the importance of complementing the pole figure analysis with an analysis of the deformation modes activity. A characteristic advantage of the VPSC formulation over the classical polycrystal models is that systems with lower CRSS tend to accommodate more deformation than systems with high CRSS: this is achieved without sacrificing compatibility, which is built in the model in an average sense. As a consequence, an average of three systems per grain suffice to accommodate deformation, the singlecrystal yield surface does not have to be closed, and the resultant textures show more spread.

The main feature of self-consistent models is the existence of an interaction equation which linearly links stress and strain rate in each grain with the equivalent magnitudes in the polycrystal. The coupling depends upon the relative anisotropies of grain and matrix, and also upon the rate sensitivity exponent assumed. Our formulation is based on a tangent viscoplastic approximation and in Section 2 we show that it gives a more compliant matrix when $n$ increases, so resembling the result of a lower bound approach. We also show that if the secant (instead of the tangent) compliance is used, a stiffer matrix (and so an upperbound-type result) is obtained for large $n$. Since both the tangent and the secant approaches are just a way around a highly non-linear problem, this work may be regarded in one of two ways: either as relevant to a fictitious material with well-defined properties similar to those of the real material, or alternatively as relevant 


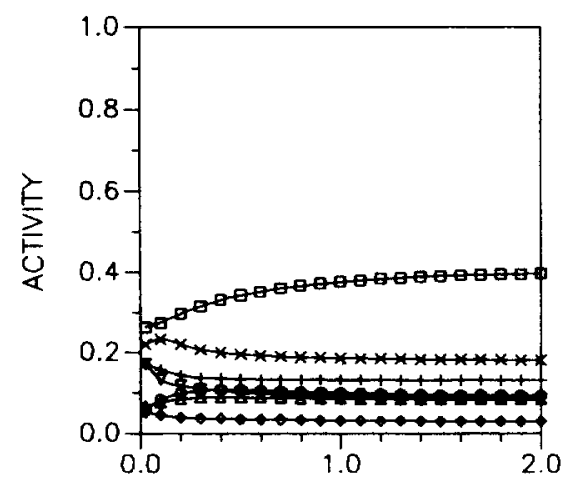

(a)

TRUE STRAIN

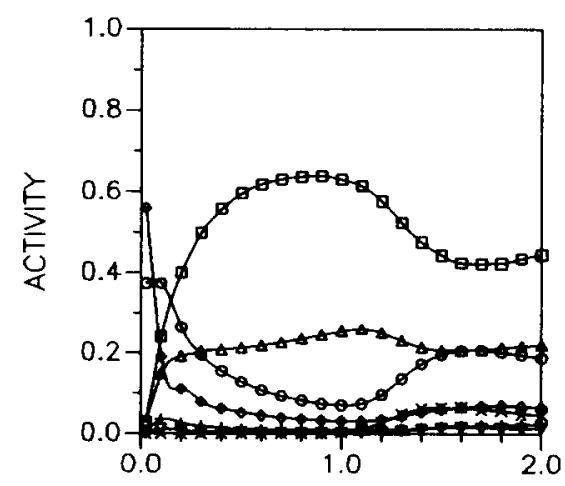

(b)

TRUE STRAIN

Fig. 10. Relative contribution of each mode to the total deformation as a function of strain for the $\alpha$-U case using $(a)$ the FC and (b) the VPSC formulation: $O,(010)[100]$ slip; $\square,(110)[110]$ slip; $\diamond, \quad(001)[100]$ slip; $\diamond, \quad(130)[310]$ slip; $\quad \nabla, \quad(172)[312]$ slip; ,$+(112)[372]$ slip; $\times,(197)[512]$ slip.

to the latter with an uncertainty introduced by the linearization of the constitutive behaviour. Sometimes, such an "effective" representation may be supported by experimental evidence, as in the case of brass discussed in Section 3.2 of this work. The VPSC scheme is appealing in this respect because it provides a formal framework to modify the strength of the interaction, where the relative stiffness of grain and matrix is still accounted for and where stress and strain averages are kept consistent with the overall boundary conditions.

A word is in order at this point concerning the treatment of twinning. Although the VFT scheme represents an improvement in the treatment of the twinned fractions and can simultaneously tackle the reorientation due to slip, there are some aspects of twinning which are not addressed by the present formulation. One of them is the stress relaxation associated with twin activation: in materials that do not twin, once a grain starts yielding, the stress remains on the yield surface and most of the subsequent deformation is plastic. When twins are active, however, a stress relaxation takes place that modifies substantially the stress in the grain and its interaction with the matrix. Account- ing for this feature would require us to include elastic effects in the self-consistent polycrystal formulation, along the lines suggested recently by Kouddane et al. [28] and Turner and Tomé [29]. Another missing feature is the fact that, while the reoriented fractions are subsequently treated as independent of the grain where they originated, we are aware that the presence of twins may influence the subsequent plastic response of the grain. The afore-mentioned limitations may be responsible for the discrepancies between the predicted and the experimental textures in the case of $\alpha$-U. As a partial conclusion, we believe that the present modelling of twinning in polycrystal codes is still crude and that there is room for improvements.

\section{References}

1 G. I. Taylor. J. Inst. Met., $62(1938) 307$.

2 J.F.W. Bishop and R. Hill, Philos. Mag., 42 (1951) 414.

3 H. Honneff and H. Mecking, in S. Nagashima (ed.), Proc. oth Int. Conf. on Texture of Materials, Iron and Steel Inst. Japan, 1981, p. 347.

4 A. Molinari, G. Canova and S. Ahzi, Acta Metall., 35 (1987) 2983.

5 P. Van Houtte, Acta Metall., 26 (1978) 591.

6 C. N. Tomé, H.-R. Wenk, G. R. Canova and U. F. Kocks, J. Geophys. Res., 96 (B7)(1991) 11865.

7 C. N. Tomé, G. R. Canova, S. Ahzi and A. Molinari, in J. S. Kallend and G. Gottstein (eds.), Proc. Int. Conf. on Texture of Materials, The Metallurgical Society, Warrendale, PA. 1988, p. 395.

8 R A. Lebensohn and C. N. Tomé. Textures Microstructures, I4-18(1991)959.

9 C. N. Tomé, R. A. Lebensohn and U. F. Kocks, Acta Metall. Mater., $39(1991) 2667$.

10 R. A. Lebensohn and C. N. Tomé, Acta Metall. Mater., 41 (1993) 2611.

11 R. A. Lebensohn and C. N. Tomé, Philos. Mag. A, 67 (1993) 187.

12 J. W. Hutchinson, Proc. R. Soc. London, Ser. A, 348 (1976) 101 .

13 T. Mura, Micromechanics of Defects in Solids, MartinusNijhoff, The Hague, 1987.

14 M. Berveiller and A. Zaoui, in Comportements Rhéologiques et Structure des Materiaux, Proc. 15th Colloq. of GFR, Paris, Groupe Français de Rheologie, p. 175.

15 E. Kroner, Acta Metall., 9(1961) 155.

16 B. Budiansky and T. T. Wu, Proc. 4th Congr. on Applied Mechanics, 1962, p. 1175.

17 D. Charquet, E. Alheritiere and G. Blanc, Zirconium in the nuclear industry, Am. Soc. Test. Mater., Spec. Tech. Publ., $939(1987) 663$.

18 E. Tenkhoff, Metall. Trans. A, $9(1978) 1401$.

19 A. Akhtar, J. Nucl. Mater., $47(1973) 79$.

20 A. Pochettino, N. Gannio, C. Vial Edwards and R. Penelle, Scr. Metall. Mater., 27(1992) 1859.

21 R. G. Ballinger, The Anisotropic Behaviour of Zircaloy-2, Garland, New York, 1979.

22 T. Leffers and D. J. Jensen, Textures Microstructures, 14-19 (1991) 933

23 H. Hu, P. R. Sperry and P. A. Beck. Trans. Metall. Soc. AIME, I94 (1952)76. 
24 T. Takeshita, C. N. Tomé, H.-R. Wenk and U. F. Kocks, J. Geophys. Res., 92 (1987) 12917.

25 J. H. Gittus, Uranium, Butterworths, London, 1963.

26 P. R. Morris, Proc. 1st Int. Conf. on Texture of Materials, 1971, p. 87.

27 A. D. Rollett, in T. C. Lowe, A. D. Rollett, P. S. Follansbee and G. S. Daehn (eds.), Modeling the Deformation of Crystal- line Solids, The Metallurgical Society, Warrendale, PA, 1991 , p. 361.

28 R. Kouddane, A. Molinari and G. R. Canova, in C. Teodosiu, J. L. Raphanel and F. Sidoroff (eds.), Large Plastic Deformations: Fundamentals and Applications, Balkama, Rotterdam, 1993.

29 P. A. Turner and C. N. Tomé, J. Mech. Phys. Solids, in press. 